\title{
The intelligent distributed acoustic sensor (withdrawal notice)
}

\section{Tom Parker, Arran Gillies, Sergey Shatalin, Mahmoud Farhadiroushan}

Tom R. Parker, Arran Gillies, Sergey V. Shatalin, Mahmoud Farhadiroushan, "The intelligent distributed acoustic sensor (withdrawal notice)," Proc. SPIE 9157, 23rd International Conference on Optical Fibre Sensors, 9157D8 (2 June 2014); doi: 10.1117/12.2068026

SPIE Event: OFS2014 23rd International Conference on Optical Fiber Sensors, 2014, Santander, Spain 


\section{The intelligent distributed acoustic sensor (withdrawal notice)}

Proc. SPIE 9157, 9157D2 (2014); http://dx.doi.org/10.1117/12.2068026

Online Publication Date: 2 June 2014

Withdrawn: 18 June 2014

Conference Date: 2-6 June 2014

Conference Location: Santander, Spain

Conference Title: 3rd International Conference on Optical Fibre Sensors

Conference Chair: José Miguel López-Higuera, Julian Jones, Manuel López-Amo, José Luis Santos

M. Farhadiroushan, A. Gilles, T. R. Parker, and S. V. Shatalin

Silixa Ltd. (United Kingdom)

This paper has been withdrawn. The following nearly identical paper is available in this conference proceedings: M. Farhadiroushan, A. Gilles, T. R. Parker, and S. V. Shatalin, " The intelligent distributed acoustic sensor," Proc. SPIE 9157, 23rd International Conference on Optical Fibre Sensors, 915730 (June 2, 2014); doi:10.1117/12.2064889. 